\title{
Viscosity and transport in a model fragile metallic glass
}

\author{
P. M. Derlet $\odot,{ }^{1,}{ }^{*}$ H. Bocquet, ${ }^{1,2}$ and R. Maaß $\odot^{3,4}$ \\ ${ }^{1}$ Condensed Matter Theory Group, Paul Scherrer Institut, CH-5232 Villigen PSI, Switzerland \\ ${ }^{2}$ Department of Mechanical Engineering, EPFL, CH-1015 Lausanne, Switzerland \\ ${ }^{3}$ Federal Institute of Materials Research and Testing (BAM), Unter den Eichen 87, Berlin 12205, Germany \\ ${ }^{4}$ Department of Materials Science and Engineering, University of Illinois at Urbana Champaign, \\ 1304 West Green Street, Urbana, Illinois 61801, USA
}

(Received 24 June 2021; accepted 8 December 2021; published 22 December 2021)

\begin{abstract}
How thermally activated structural excitations quantitatively mediate transport and microplasticity in a model binary glass at the microsecond timescale is revealed using atomistic simulation. These local excitations, involving a stringlike sequence of atomic displacements, admit a far-field shear-stress signature and underlie the transport of free-volume and bond geometry. Such transport is found to correspond to the evolution of a disclination network describing the spatial connectivity of topologically distinct bonding environments, demonstrating the important role of geometrical frustration in both glass structure and its underlying dynamics.
\end{abstract}

DOI: 10.1103/PhysRevMaterials.5.125601

\section{INTRODUCTION}

The nature of a deeply undercooled metal-alloy liquid and its transition to a bulk metallic glass may be characterized by local structural motifs quite different from both the equilibrium liquid and solid phase [1], and whose fluctuations become increasingly heterogeneous in both space and time as the temperature reduces [2]. While the larger-scale collective processes eventually freeze-out, there still exist fast and more localized structural fluctuations which mediate relaxation and plasticity of the amorphous metallic solid. Quantifying the properties and constraints of such dynamics is therefore essential for a fundamental understanding of the property-structure relationship for both old, and new, engineering relevant metallic glasses, and glassy solids in general [3].

As a structural measure, free-volume has played an important role in the phenomenology of undercooled metallic liquids and bulk metallic glasses [4]. For simple liquids, Turnbull and Cohen [5,6] hypothesized transport, and thus flow, was facilitated by ballistic particle rearrangements sometimes resulting in the creation of local free-volume into which a neighboring particle could enter. Spaepen [7] extended this to bulk-metallic glasses by including a barrier energy to freevolume migration, and treating the frozen-in free-volume as a state variable characterizing the glassy structure and its plastic flow properties. Here it was envisioned that an applied shear stress could cause a local dilation and the creation of new free-volume. This was further developed by Argon [8], who replaced localized free-volume migration with a local shear transformation (ST). High strain rate atomistic simulation has given great insight into the athermal variant of Argon's thermally activated ST [9] - the shear transformation zone [10,11] (STZ). On the other hand, simulation work under zero load has also revealed glassy structural changes mediated by ther-

*peter.derlet@psi.ch mally activated cooperative atomic displacements [12-16], here referred to as localized structural excitations (LSEs).

The core structural change of an LSE involves a sequence of atoms changing their position with a neighbor (inset of Fig. 1). Such stringlike excitations also occur in the undercooled liquid regime [18-22] and are fundamental to the drastic changes in transport occurring during the glass transition [2]. Within the amorphous-solid regime, LSEs constitute the elementary excitations of the glassy structure, where potential energy landscape (PEL) exploration algorithms were able to identify the energy barriers [23-26] and via harmonictransition state theory give estimates of the corresponding attempt rate [27]. LSEs are to be distinguished from STZs which are only active under load, correspond to stress induced inflection points of the PEL, and tend to be more spatially localized, not having the LSE stringlike geometry. Finite temperature simulations demonstrate that LSE activity occurs in regions lacking icosahedral content (Fig. 1) and results in structural relaxation [28,29], stress relaxation [30], and microplasticity [31]. The separation of structure into regions of icosahedral and nonicosahedral content is a general phenomenon [17,32-35] fundamentally connected to the level of geometrical constraint and frustration occurring within the amorphous solid [36-40]. How LSE occurrence is related to this geometrical frustration, its relation to free-volume content, and how it mediates transport and viscosity below the glass transition regime, remain quantitatively unknown, and will be addressed in the present work.

To this end, we generalize the work of Spaepen [7] and Argon [8], and develop a phenomenological framework to study LSEs based on a distribution of activated processes, each characterized by activation parameters, a far-field stress/strain, and a near-field bond-length displacement. An inverse relationship between the instantaneous diffusion $D$ and viscosity $\eta$ parameters is established, which contains no explicit temperature dependence but is proportional to the density of possible LSEs. Here the terminology of instantaneous is used 


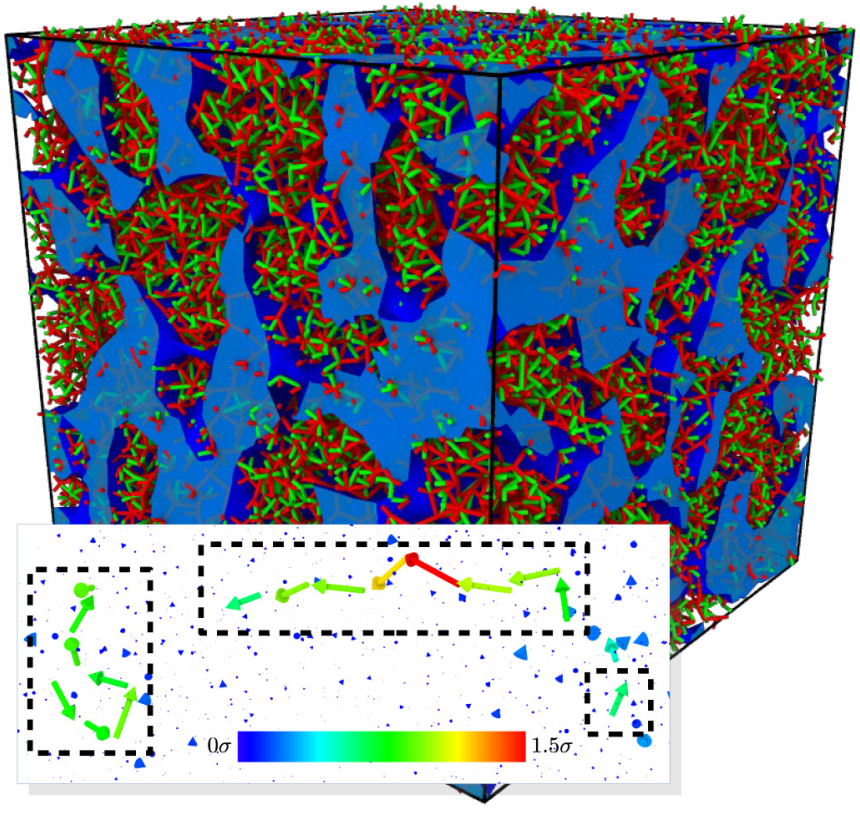

FIG. 1. Glassy atomic configuration visualized through a nearest neighbor bond network in which each bond is colored according to its bond order (the number of common neighbors shared by the two atoms forming the bond). Here red bonds represent sixfold and green bonds fourfold bond orders, whereas fivefold bonds are not visualized. An atom containing only 12 fivefold bonds has a local icosahedral structure, whereas an atom containing also four- and sixfold bond orders is seen as a defected icosahedral environment. From this perspective, the latter form a disclination network along which the fivefold icosahedral symmetry has been broken. The visualized isosurface encloses atoms which have (mainly) icosahedral and Frank-Kasper structural motifs, regions that only contain sixfold disclination lines. Outside this region, both fourfold and sixfold disclination structures are seen, reflecting both Frank-Kasper and non-Frank-Kasper structural motifs, and an excited structural state for which dissipation occurs via thermally activated localizes structural excitations-stringlike cooperative activity visualized through atomic displacements (inset, where the color of the arrows indicate the corresponding displacement magnitude). Data are derived from atomistic simulations performed at $0.8 T_{\mathrm{g}}$ and the structural analysis is performed using a modified Voronoi tesselation described in Ref. [17].

to emphasize the dependence of such quantities over the (short) timescale over which they are measured. Values of both $D$ and $\eta$ are obtained from microsecond long atomistic simulations revealing LSEs to be considerably more numerous than that suggested by free-volume theories. Within this phenomenology we fully characterize the LSEs, finding their occurrence entails free-volume transport which on average is volume conservative and thus nondilative, resulting in a far-field shear stress signature as suggested by Eshelby [41]. Concurrent to this is the transport of coordination and bond order which satisfies the topologically allowed deviations away from minimally frustrated local icosahedral motifs. Thus an important link between glass structure and the excitations mediating transport and dissipation, and how this leads to a less frustrated structure and emergent spatial heterogeneities is quantitatively established.
In Sec. II a phenomenology relating the microscopic properties of LSEs to bulk transport and microplasticity is developed, as is a viscoelastic model to describe the microplasticity seen in Ref. [31], the latter of which will allow for an estimation of the instantaneous viscosity. This is followed by Sec. III which applies these phenomenologies to the atomistic data of Ref. [31] to determine the key average properties of LSEs. The final section, Sec. IV, provides a brief summary of the work and some concluding remarks.

\section{PHENOMENOLOGY}

\section{A. Localized structural excitations (LSEs)}

Acknowledging the complexity of the glass PEL, we consider a population of LSEs that mediate thermally activated relaxation, and when under a load $\sigma$, mediate thermally activated plastic flow. Within a volume $V$ of $N$ atoms, $M=$ $\rho_{\text {LSE }} V$, LSEs are assumed to exist, each of which has a probability of occurrence within a time interval $\Delta t$ equal to

$$
\Gamma_{i} \Delta t=\Delta t v_{i} \exp \left[-\beta E_{i}(\sigma)\right],
$$

where $\beta=\left(k_{\mathrm{B}} T\right)^{-1}$, and $E_{i}(\sigma)$ and $v_{i}$ are the shear stress dependent barrier energy and attempt rate for the $i$ th LSE with occurrence rate $\Gamma_{i}$. Defining an activation volume $\Omega_{0 i}$ via $E_{i}(\sigma) \approx E_{0 i}-\sigma \Omega_{0 i}$, this becomes

$$
\begin{aligned}
\Gamma_{i} \Delta t & \approx \Delta t v_{i} \exp \left[-\beta E_{0 i}\right]\left(1+\beta \Omega_{0 i} \sigma\right) \\
& =\Gamma_{0 i} \Delta t\left(1+\beta \Omega_{0 i} \sigma\right)
\end{aligned}
$$

for the regime of $\beta \Omega_{0 i} \sigma \ll 1$.

The $i$ th LSE is therefore characterized by an energy barrier $E_{0 i}$, activation volume $\Omega_{0 i}$, and attempt rate $v_{i}$. PEL exploration algorithms $[23,24]$ show that for a well relaxed sample, the positive value distributions of $E_{0 i}$ and $v_{i}$ are unimodal and approach zero at small values, $v_{0 i}$ is weakly correlated with $E_{0 i}$ [27], as is $\Omega_{0 i}$ which can be positive or negative [25]. LSEs are assumed to affect the surrounding glassy structure through their far-field plastic shear strain $\Delta \varepsilon_{i}(\sim 1 / V$, due to the general scaling properties of strain with volume), and by core LSE atom displacements characterized by a mean-square displacement $\Delta r_{i}^{2}$ that underlies transport.

Within the volume $V$, the average number of LSEs occurring in $\Delta t$ is given by

$$
\Gamma \Delta t=\sum_{i=1}^{M} \Gamma_{i} \Delta t=M\langle\Gamma\rangle \Delta t=\rho_{\mathrm{LSE}} V\langle\Gamma\rangle \Delta t .
$$

The corresponding average plastic strain due to an applied stress $\sigma$ occurring within this time interval and volume $V$ is then

$$
\begin{aligned}
\left\langle\Delta \varepsilon_{\mathrm{p}}\right\rangle & =\sum_{i=1}^{M} \Gamma_{i} \Delta \varepsilon_{i} \Delta t \\
& =M\langle\Gamma \Delta \varepsilon\rangle \Delta t \\
& \approx M\left\langle\Gamma_{0}\left(1+\beta \Omega_{0} \sigma\right) \Delta \varepsilon\right\rangle \Delta t \\
& =M\left\langle\Gamma_{0}\right\rangle \Delta t \beta\left\langle\Delta \varepsilon \Omega_{0}\right\rangle \sigma .
\end{aligned}
$$

In the last equality of Eq. (4), $\langle\Delta \varepsilon\rangle=0$ is assumed, entailing the distribution of far-field shear strains spans all possible slip 
directions reflecting the complexity of the glassy structure and its possible excitations.

The average total atomic (cumulative) displacement under zero load can be derived in a similar manner:

$$
\begin{aligned}
\left\langle\Delta r_{\text {Total }}^{2}\right\rangle & =\sum_{i=1}^{M} \Gamma_{i} \Delta r_{i}^{2} \Delta t \\
& =M\left\langle\Gamma \Delta r^{2}\right\rangle \Delta t \\
& =M\left\langle\Gamma_{0}\right\rangle \Delta t\left\langle\Delta r^{2}\right\rangle,
\end{aligned}
$$

where in the last equality, the LSE mean-square displacement is assumed to be uncorrelated with the LSE barrier energy and the attempt rate.

Defining the instantaneous viscosity via $\left\langle\Delta \varepsilon_{\mathrm{p}}\right\rangle / \Delta t \doteq$ $\sigma / \eta$ and the instantaneous diffusion constant via $D \doteq$ $\left\langle\Delta r_{\text {Total }}^{2}\right\rangle /(6 N \Delta t)$ gives

$$
\eta \doteq\left(\rho_{\mathrm{LSE}} \frac{V\left\langle\Delta \varepsilon \Omega_{0}\right\rangle}{k_{\mathrm{B}} T}\left\langle\Gamma_{0}\right\rangle\right)^{-1}
$$

and

$$
D \doteq \frac{1}{6} \frac{\rho_{\mathrm{LSE}}}{\rho_{\text {Atom }}}\left\langle\Delta r^{2}\right\rangle\left\langle\Gamma_{0}\right\rangle .
$$

Both equations have used $M=\rho_{\mathrm{LSE}} V$.

Assuming the fundamental (Maxwell) timescale is also related to the viscosity through $\left\langle\Gamma_{0}\right\rangle^{-1}=\eta / G$, where $G$ is an appropriate shear modulus, Eqs. (6) and (7) give

$$
D \eta=\frac{1}{6} \frac{\rho_{\mathrm{LSE}}}{\rho_{\text {Atom }}} G\left\langle\Delta r^{2}\right\rangle .
$$

The imposition of $\left\langle\Gamma_{0}\right\rangle^{-1}=\eta / G$ entails LSE occurrence does not spontaneously induce further significant LSE activity at the timescale of $\Delta t$. This may be seen by the substitution of $\left\langle\Gamma_{0}\right\rangle^{-1}=\eta / G$ into Eq. (6), which gives

$$
\beta\left\langle\Delta \varepsilon \Omega_{0}\right\rangle G=\frac{1}{M} \sim \frac{1}{V} .
$$

The left-hand-side of the above can be viewed as the second term in the last equality of Eq. (2), showing the effect on the LSE activity due to the change in stress arising from an LSE occurrence will be negligible.

The above derivations assume the statistical independence of LSE activity. This assumption becomes invalid at a lower "cooperative" length scale where the system cannot independently sample all LSE transition paths. The leading order effect of this may be taken into account by dividing the prefactors $v_{i}$ by $M_{\text {coop }}$, the characteristic number of LSEs within a cooperative volume [42]. It is also noted that all $\langle\cdot\rangle$ quantities will depend implicitly on $\Delta t$ and temperature. For a small enough $\Delta t$ or low enough temperature, only a subset of the $M, \Gamma_{i} \Delta t$ will be thermally accessible, a regime of statistics for the determination of $\langle\cdot\rangle$ tending towards the extreme-value asymptote [43]. This emphasizes that all phenomenological quantities (and their assumed lack of correlation) are representative of the timescale $\Delta t$ and temperature at which they are measured. It is because of this that the parameters $D$ and $\eta$ are referred to as instantaneous quantities.

Because Eq. (8) has no explicit temperature dependence, it is tempting to interpret it as a breakdown of the
Stokes-Einstein relation seen both experimentally [44] and computationally [45] for temperatures up to and beyond the liquidus temperature. Such a breakdown is related to a separation of the bulk viscous and bulk diffusion timescales, which is not the case for our values of $D$ and $\eta$-instantaneous quantities that here do not capture the long-time collective properties of LSE activity.

\section{B. Microplasticity}

Motivated by the microplasticity simulations of Ref. [31], a classical two phase elastic/viscoelastic model is developed. An isostrain scenario is considered in which the global stress is a volume-fraction weighted sum of the characteristic stress of a purely elastic region (E) and a viscoelastic region (VE) region: $\varepsilon_{\mathrm{E}}=\varepsilon_{\mathrm{VE}}=\varepsilon$ and $\sigma=\Omega_{\mathrm{E}} \sigma_{\mathrm{E}}+\Omega_{\mathrm{VE}} \sigma_{\mathrm{VE}}$. Here $\varepsilon$ is the global strain state and $\Omega_{\mathrm{E} / \mathrm{VE}}$ and $\sigma_{\mathrm{E} / \mathrm{VE}}$ are the volume fractions and characteristic stress of the elastic/viscoelastic regions. Such a partition of stress is seen in the atomistic simulations (see Sec. 3.1 of Ref. [31]). By definition, the elastic region experiences only an elastic response, given by $\sigma_{\mathrm{E}}=$ $G \varepsilon$, where $G$ is a global shear modulus applicable to both the elastic and viscoelastic regions. On the other hand, the viscoelastic region experiences both an elastic and viscoplastic response, and is characterized by a linear decomposition of the applied strain, $\varepsilon_{\mathrm{VE}}=\varepsilon_{\mathrm{VE}}^{\text {elastic }}+\varepsilon_{\mathrm{VE}}^{\text {plastic }}=\varepsilon$ or, in terms of strain rate, $\dot{\varepsilon}_{\mathrm{VE}}^{\text {elastic }}+\dot{\varepsilon}_{\mathrm{VE}}^{\text {plastic }}=\dot{\varepsilon}$. The elastic contribution to the strain rate is given by $\dot{\varepsilon}_{\mathrm{VE}}^{\text {elastic }}=\dot{\sigma}_{\mathrm{VE}} / G$, whereas the plastic contribution is written as $\dot{\varepsilon}_{\mathrm{VE}}^{\text {plastic }} \doteq \sigma_{\mathrm{VE}} / \eta_{\mathrm{VE}}(t)$ defining a time-dependent instantaneous viscosity for the viscoelastic region.

Together, the above considerations give

$$
\sigma(t)+\frac{\eta_{\mathrm{VE}}(t)}{G} \dot{\sigma}(t)=\Omega_{\mathrm{E}} G \varepsilon(t)+\eta_{\mathrm{VE}}(t) \dot{\varepsilon}(t),
$$

which has the general form of a Maxwell representation of a standard linear solid description of viscoelasticity. Indeed, in the limit of a vanishing purely elastic region, $\Omega_{\mathrm{E}} \rightarrow 0$ and $\eta_{\mathrm{VE}}(t)=\eta_{0}$, the above corresponds to the well known Maxwell equation for viscoelasticity.

For the initial condition $\sigma(t=0)=0$ and a fixed applied strain rate $\dot{\varepsilon}(t)=\dot{\varepsilon}_{\text {app }}$, Eq. (10) has the solution:

$$
\sigma(t)=\dot{\varepsilon}_{\mathrm{app}} G \int_{0}^{t} d t^{\prime} e^{-G F\left(t^{\prime}, t\right)}\left(1+\frac{G \Omega_{\mathrm{E}} t^{\prime}}{\eta_{\mathrm{NI}}\left(t^{\prime}\right)}\right),
$$

where

$$
F\left(t_{0}, t_{1}\right)=\int_{t_{0}}^{t_{1}} d t^{\prime \prime} \frac{1}{\eta_{\mathrm{VE}}\left(t^{\prime \prime}\right)} .
$$

Such a solution will be used to describe the microplastic response of the elastic loading regime under constant strain rate conditions.

\section{LSE PROPERTIES FROM ATOMISTIC SIMULATION}

\section{A. Methodology}

The zero-load data set taken from Ref. [31] represents 2 $\mu$ s of physical simulation of a model Lennard-Jones binary system [46], using a 50:50 composition of small to large atoms. The initial configuration is the same configuration 
used for the loading simulations of Ref. [31] and numbers 32000 atoms. The simulations were performed at fixed zero pressure under isotropic volume fluctuations, at a temperature of $0.8 T_{\mathrm{g}}$, where $T_{\mathrm{g}}$ is the fictive glass transition temperature [31]. During the simulation, atomic data was stored every $1 \mathrm{~ns}$ resulting in 2000 configurations spanning $2 \mu \mathrm{s}$. For each configuration, a conjugate gradient relaxation was performed to find a local minimum of the potential energy resulting in an inherent state of the system. The volume of each configuration was kept fixed during this relaxation. Thus any structural deviation must involve the system transiting from one local potential energy minimum to another. For the calculation of the change in global stress, the simulation cells of all 2000 configurations were rescaled to that of the initial configuration allowing for a meaningful calculation of the dilatational stress due to LSE activity while negligibly affecting the shear stress components.

All distances and energies are measured in terms of the respective Lennard-Jones parameters $\sigma$ and $\varepsilon$. For the definition of time, see Ref. [31]. For a discussion on using a different ratio of atomic sizes and/or for compositions deviating from the used 50:50, see Refs. [17,29]. Bond-order analysis is done via a modified Voronoi tessellation that is better able to tessellate a given atomic configuration in terms of minimally distorted tetrahedra $[17,47]$.

\section{B. Instantaneous diffusion coefficient and viscosity}

Taking $\Delta t$ to be $1 \mathrm{~ns}$, we study LSE activity using the zero-load atomistic simulation data set of Ref. [31]. Core LSE atoms may be identified as those which displace more than 0.6 of a bond length within $\Delta t$ (see inset of Fig. 1). This numerical value is the minimum of the Van Hove self-correlation function separating bond length from sub-bond-length displacements (see Fig. 7(c) of Ref. [31]). Figure 2(a) plots the number of detected LSEs, $N_{\mathrm{LSE}}$, as a function of time, showing the number decreases from 25 to 10 as the structure relaxes (see Fig. 4(a) of Ref. [31]). Such unit LSE data is obtained by a nearest-neighbor cluster analysis of all LSE core atoms. Figure 2(b) gives a scatter plot between $N_{\mathrm{LSE}}$ and the total mean-square disaplacement due to only the core LSE atoms, $\left\langle\Delta r_{\text {Total }}^{2}\right\rangle$. A good linear correlation is evident, giving $\left\langle\Delta r^{2}\right\rangle \approx(2.40 \pm 0.01) \sigma^{2}$.

The outlier points associated with the larger values of $N_{\mathrm{LSE}}$ seen in Fig. 2(a), and also the values at the earliest times, generally correspond to the outliers in Fig. 2(b) that deviate from the linear correlation seen for $N_{\mathrm{LSE}}<20$. This observation represents a good example of the dependence of the current results on timescale $(\Delta t)$ and the absolute time at which the analysis is performed (the microstructural state). At $\Delta t=$ $1 \mathrm{~ns}$, the results of our analysis reveal that the average meansquare displacement of an LSE is $\left\langle\Delta r^{2}\right\rangle \approx(2.40 \pm 0.01) \sigma^{2}$. When inspecting the displacements of individual core LSE atoms, this value reflects several atoms changing their position approximately a bond length (see inset in Fig. 1). Inspection of the spatial autocorrelation (Van Hove) function at $\Delta t=1 \mathrm{~ns}$ (see Fig. 7(c) of Ref. [31]) reveals this to be the most probable outcome. However, such a correlation function is not Gaussian, having a fat-tail distribution for larger displacements. Increased sampling $\left(N_{\mathrm{LSE}}\right)$ of this distribution will result in

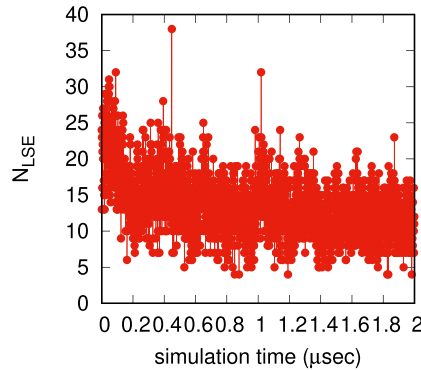

(a)

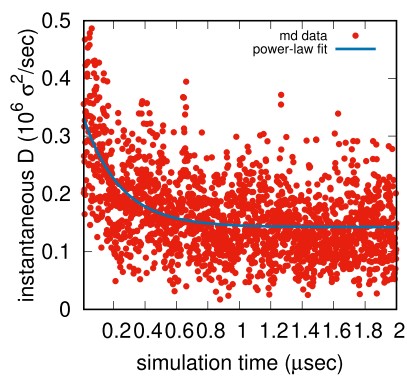

(c)

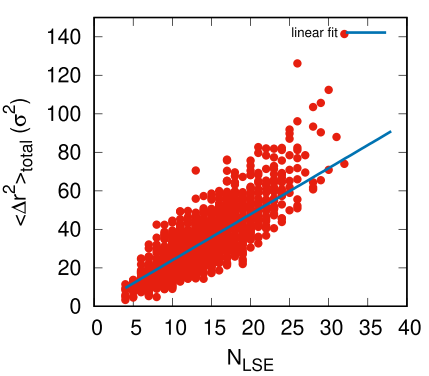

(b)

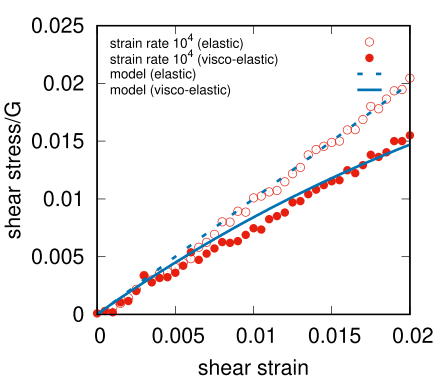

(d)
FIG. 2. (a) LSE number evolution and (b) corresponding scatter plot of LSE number with LSE mean-square displacement. (c) Plot of instantaneous diffusion as a function of time derived from the zero-load data. (d) Stress-strain response for a strain rate of $10^{4} / \mathrm{s}$ decomposed in the contribution arising from the elastic (icosahedral atoms) and the viscoelastic regions. The solid lines represent the optimal fit of the developed two-phase elastic/viscoelastic model.

a larger mean value which deviates more and more from the most probable displacement. This observation reflects itself in the observed nonlinear trend seen in Fig. 2(b).

What is the origin of increased LSE activity $\left(N_{\mathrm{LSE}}\right)$ over the chosen time interval? For the earlier times, the glassy structure is less relaxed admitting increased LSE activity, however there also exist rare increases in $N_{\text {LSE }}$ at later times suggesting some form of intermittent correlated LSE activity that would increasingly affect the average LSE properties when $\Delta t$ is increased. This again emphasizes the instantaneous nature of the transport and dissipation processes being presently studied.

Dividing $\left\langle\Delta r_{\text {Total }}^{2}\right\rangle$ by $6 N \Delta t$ gives an estimate of $D(t)$, which because of the varying levels of LSE activity [Fig. 2(a)] is now time dependent. Figure 2(c) plots the evolution of $D(t)$ over the $2 \mu$ s of available data, and also a fit using $D(t)=D_{0}+D_{1} \exp \left(-t / t_{0}\right)$ with $D_{1}=0.14 \sigma^{2} / \mu \mathrm{s}, D_{2}=$ $0.197 \sigma^{2} / \mu \mathrm{s}$, and $t_{0}=0.23 \mu \mathrm{s}$. Due to the strong scatter in atomistic data, the chosen mathematical form is one of many able to reproduce the average trend seen in $D(t)$. Equation (8) indicates that if $\eta(t)$ is also known then $\rho_{\mathrm{LSE}}$ can be determined. To obtain an estimate of $\eta(t)$ the two-phase model developed in Sec. II B is fitted to the deformation simulations of Ref. [31]. In this past work the slowest strain rate considered was $10^{4} / \mathrm{s}$. Up to a strain of 0.02 , this deformation spans the $2 \mu$ s of physical time covered by the zero-load simulations used for the determination of LSE properties and therefore $D(t)$ in Fig. 2(c). Thus the present fitting procedure focuses on an optimal fit of Eq. (11) to the $10^{4} / \mathrm{s}$ microplasticity data, and in particular the degree of elastic softening seen in the viscoelastic component of the system's stress response. 
In the work of Ref. [31], the purely elastic region was identified as the system-spanning network of local icosahedral environments of the smaller atom, the rational being that for the smaller atom, this structural motif is minimally frustrated. However, for the larger atom, it is the Frank-Kasper environments that are minimally frustrated. Detailed inspection of the system spanning region (enclosed by the surface in Fig. 1) reveals the inner structure is dominated by icosahedral regions with a small population of the Frank-Kasper structural motifs. Despite the presence of the latter, we consider the fractional volume of the purely elastically deforming region as consisting of only those atoms that are icosahedrally coordinated. When doing this, from the $\approx 0.2 \%$ of atoms which belong to a core LSE structure within $\Delta t, 95 \%$ of these occur outside this as-defined elastically deforming region. Indeed, including such Frank-Kasper motifs generally softens this nominally elastic region.

Figure 2(d) displays the simulated stress evolution during deformation of the elastic and viscoelasic regions (taken from the simulations of Ref. [31]) as well as the optimal fit arising from the viscoelastic model [Eqs. (8)-(12)] using $\Omega_{\mathrm{E}}=0.24$ and the global elastic shear stiffness $G=$ $16.5949 \varepsilon / \sigma^{3}$, which gives the estimate $\rho_{\mathrm{LSE}} / \rho_{\text {Atom }} \approx 1.1 \pm$ $0.2 \sim O(1)$. This value is many orders of magnitude larger than that anticipated by free-volume theory which gives the per site probability as $\exp \left(-\gamma v^{*} / v_{\mathrm{f}}\right)$ for which $\gamma v^{*} / v_{\mathrm{f}}$ is typically $O(10)$. Here $\gamma v^{*}$ is related to the particle volume and $v_{\mathrm{f}}$ to the average free-volume available to a particle. The obtained instantaneous viscosity for the viscoelastic region, expressed as $\eta / G$, is in the range 1-3 $\mu$ s which is comparable to the timescale for strain recovery seen in Ref. [31].

\section{Far-field LSE properties}

The theoretical work of Eshelby on plastic inclusions [41] suggests the resulting far-field stress/strain signature of an LSE will have a quadrapolar form and decay as $r^{-3}$. While the quadrapolar structure has been observed for stress-driven STZs via simulation [48] and experimentally via deformed colloidal glasses [49], it has yet to be for thermally activated LSEs. Because of the fixed shear strain conditions, we focus on the LSE stress signature and calculate the local atomic stress differences between two configurations separated by $\Delta t$. The corresponding change in stress arises from the 10-25 LSEs that normally occur during $\Delta t$. These local stresses are summed for the core LSE atoms and those atoms at most a distance $\delta$ away. Figure 3(a) plots the resulting von Mises stress invariant as a function of $\delta$, for 20 pairs of configurations. For $\delta<1.25 \sigma$ the local von Mises stress of the core LSE atoms fluctuates strongly. In the intermediate range, $1.25 \sigma<\delta<10 \sigma$, a clearer trend emerges.

Insight into this intermediate regime is given by considering an idealized distribution of internal stress variations due to multiple LSE creations that together contribute to a global stress change. Indeed, the change in stress over a volume $V$ is given by

$$
\Delta \sigma^{\mu \nu}=\frac{1}{V} \int_{V} d^{3} \mathbf{r} \Delta \sigma^{\mu \nu}(\mathbf{r})=\frac{1}{V} \sum_{i} V_{i} \Delta \sigma_{i}^{\mu \nu}
$$

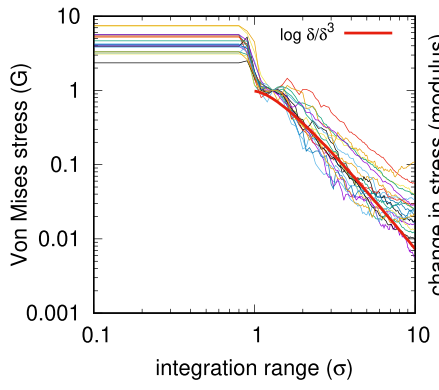

(a) (b)

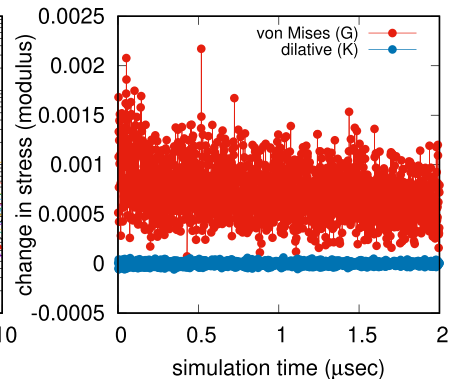

FIG. 3. (a) Plot of 20 cumulative von Mises stresses as a function of distance $\delta$ away from any core LSE atom. To enhance the observed $\log \delta / \delta^{3}$ quadrapolar scaling, the data have been shifted and when needed reflected such that all curves are equal at $\delta=1.25 \sigma$. (b) Resulting change in dilative and von Mises stress due to LSE activity. Stresses in (a) and (b) are given in terms of the bulk $(K)$ and shear $(G)$ moduli.

Here the first equality is with respect to a continuous function $\Delta \sigma^{\mu \nu}(\mathbf{r})$, representing the internal stress change of the system, whereas the second equality is with respect to the local atomic stress change $\Delta \sigma_{i}^{\mu \nu}$ of the $i$ th atom with atomic volume $V_{i}$. In the latter, the summation is over those atoms contained in the volume $V$, and is the expression used in atomistic simulation.

The data in Fig. 3(a) is calculated using the volume $V_{\delta}$ which is defined as all points within a distance $\delta$ from an LSE core atom. For the intermediate range of distances, $V_{\delta}$ may be approximated as a summation of nonoverlapping spherical volumes centered around each LSE core region of volume $V_{\delta}^{\prime}$. In this regime, for $M^{\prime}$ occurring LSEs, we have

$$
\Delta \sigma_{\delta}^{\mu \nu}=\frac{1}{M^{\prime} V_{\delta}^{\prime}} \sum_{i} \int_{V_{\delta}^{\prime}} d^{3} \mathbf{r} \Delta \sigma_{i}^{\mu \nu}(\mathbf{r}),
$$

where now $\Delta \sigma_{i}^{\mu \nu}(\mathbf{r})$ represents the internal stress change due to the $i$ th occurring LSE. Here each integral is centered on its LSE core region. This may be rewritten as

$$
\Delta \sigma_{\delta}^{\mu \nu}=\frac{1}{V_{\delta}^{\prime}} \int_{V_{\delta}^{\prime}} d^{3} \mathbf{r}\left\langle\Delta \sigma_{i}^{\mu \nu}(\mathbf{r})\right\rangle_{M^{\prime}}
$$

For increasing $M^{\prime},\left\langle\Delta \sigma_{i}^{\mu \nu}(\mathbf{r})\right\rangle_{M^{\prime}}$ becomes an increasingly isotropic function. Assuming a leading order quadrapolar form for the characteristic far-field shear stress results in this function scaling as $1 / r^{3}$ and therefore Eq. (14) scaling as $\log \delta / \delta^{3}$.

Inspection of Fig. 3(a) demonstrates that a $\log \delta / \delta^{3}$ scaling is compatible with the data. For $\delta>10 \sigma$ in Fig. 3(a), almost all atoms are included in the summation and the stress converges to the total change in von Mises stress due to LSE activity. Indeed, such a shear stress entirely dominates the farfield stress signature, with the dilatational component being comparatively negligible [see Fig. 3(b)]. Together, these results give strong evidence that LSE occurrence may be viewed as an Eshelby plastic shear inclusion giving theoretical support to past coarse-grained thermal theories of glass plasticity [50-54]. 
TABLE I. Average change in volume, coordination ( $Z$ ), and four, five-, and six-bond orders at start and end environments of core LSE displacements. It is noted that $\Delta N_{6}-\Delta N_{4}=\Delta Z$ is approximately satisfied.

\begin{tabular}{lcc}
\hline \hline Measure & Start & End \\
\hline & Large atoms \\
Free-volume & $0.065 \pm 0.064 \sigma^{3}$ & $-0.066 \pm 0.063 \sigma^{3}$ \\
Coordination & $1.16 \pm 1.88$ & $-1.19 \pm 1.87$ \\
$\left(\Delta N_{4}, \Delta N_{5}, \Delta N_{6}\right)$ & $(-0.25,0.60,0.87)$ & $(0.24,-0.57,-0.90)$ \\
& Small atoms & \\
Free-volume & $0.033 \pm 0.053 \sigma^{3}$ & $-0.033 \pm 0.053 \sigma^{3}$ \\
Coordination & $0.65 \pm 1.84$ & $-0.70 \pm 1.83$ \\
$\left(\Delta N_{4}, \Delta N_{5}, \Delta N_{6}\right)$ & $(-0.19,0.42,0.41)$ & $(0.19,-0.43,-0.42)$ \\
\hline \hline
\end{tabular}

\section{LSE conservative free-volume transport}

The characteristic stringlike geometry seen in Fig. 1 motivates three distinct core atomic environments: the start-point, internal, and end-point core LSE atoms. Table I lists the average change in atomic volume for the start and end points derived from the entire $2 \mu$ s zero-load data set. The local atomic volume of the start and end configurations is calculated using a modified Voronoi tesselation [17]. Table I demonstrates an increase at the start point and a comparable decrease at the end point, whereas the internal regions (not shown) entail order-of-magnitude less average changes. Thus LSEs transport free-volume antiparallel to the displacement direction, indicating a less local generalization of the free-volume migration first envisaged by Turnbull and Cohen [6]. Table I also reveals such transport to be on average conserved suggesting a minimal average far-field dilatational stress signature - a result entirely consistent with a dominant far-field shear stress signature seen in Fig. 3(b).

Since the ratio between the volume transported (Table I) and the present atomic volumes $\left(V_{\text {small }} / V_{\text {large }} \approx 0.65 / 0.78\right)$ is comparable to $\gamma v^{*} / v_{\mathrm{f}}$, there is a historical justification to refer to the transported volume as free-volume. Such free-volume content is a direct measure of glass structural relaxation $[17,29,33,55,56]$ - a process intimately connected to the creation of icosahedral content associated with the smaller atom [30]. Icosahedral content is characterized by a minimally frustrated local environment containing 12 fivefold bonds (an $n$-fold bond between two atoms has $n$ common neighbors) which has the lowest volume per atom and is largely absent of LSE activity. Deviations away from this environment facilitate the fundamental connection between coordination and free-volume, since higher coordinated environments have larger atomic volumes to accommodate the larger number of nearest neighbors. This is reflected in the concurrent transport of coordination, which Table I shows to be also on-average conservative.

\section{E. LSE-driven disclination network evolution}

To gain insight into the changes in coordination, the topologically distinct deviations from the icosahedral environment must be considered, an approach initiated by Frank and Kasper [36,57], Bernel [37], Chaudhari and Turnbull [38], and later given a mathematical foundation by Nelson $[39,40]$. These works show that (low energy) topologically distinct deviations away from the icosahedral environment consist of bonding environments defined by the number of four-, five-, and sixfold nearest-neighbor bonds $\left(N_{4}, N_{5}, N_{6}\right)$. Recent work [17] shows that up to $95 \%$ of a glassy structure quantitatively follows the predicted bonding topologies. The coordination is trivially given by $Z=$ $N_{4}+N_{5}+N_{6}$, but since the presence of a fourfold/sixfold bond entails a decrease/increase in coordination one also has $Z=12-N_{4}+N_{6}$, giving $\left(N_{4}, 12-2 N_{4}, N_{6}\right)$ and thus a change in bond order $\left(\Delta N_{4},-2 \Delta N_{4}, \Delta N_{6}\right)$ and change in coordination $\Delta Z=N_{6}-N_{4}$. Table I includes the average changes in bond order for the start- and end-point core LSE atoms, showing both relations to hold. More generally, on average, two fivefold and two sixfold bonds are transported with free-volume migration, for every fourfold bond transported in the opposite direction, which together results in the expected change in coordination.

When the defect bonds are seen as disclination segments (along which the fivefold symmetry of the icosahedron is broken), the glassy structure may be viewed as a complex network of extended disclinations whose nodal points satisfy the bonding topologies dictated by Ref. [40]- see Fig. 1. Such line defects also convey the connectivity of variations in coordination, and the present work establishes that LSE facilitate nonlocal variations in the network's nodal points and coordination. The observed stringlike geometry of these structural changes seen in a variety of past works [18-31] is therefore a direct result of these local topological bonding constraints and the line defect nature of the corresponding disclination network.

While the data of Table I suggests on-average conservative transport, this cannot be the case over long times, with relaxation tending towards lower free-volume characterized by increased icosahedral content for the smaller atoms and Frank-Kasper motifs (bonding topologies involving sixfold bonds) for the larger atoms [17,29]. In Ref. [17], structural evolution entailed an average bond-order evolution characterized by the creation of two fivefold bonds at the expense of a fourfold and sixfold bond. This trend is also seen in the present data and suggests a topological bond order relaxation pathway characterized by $(3,6, \cdot) \rightarrow(2,8, \cdot) \rightarrow(1,10, \cdot) \rightarrow$ $(0,12, \cdot)$. Such transitions are expected to underly the fluctuations away from the mean quantities shown in Table I. How such changes in bonding topology occur is not yet clear, but due to the constrained connectivity of the disclination network, atomic environments beyond the core LSE structure will play an important role again indicating the collective nature of the disclination network [17]. The growing system spanning icosahedral and Frank-Kasper motifs (also seen in material-specific model systems [17,58,59]) are thus strong candidates for the emergent heterogeneities believed to control not only the properties of the amorphous solid $[60,61]$ but also the level of fragility in the undercooled liquid and glass transition regime [62].

\section{SUMMARY AND CONCLUSIONS}

We have presented a phenomenology that relates dissipation, via an instantaneous viscosity and diffusion, to atomic- 
scale parameters that characterize the mediating thermally activated cooperative atomic displacements, also referred to as local structural excitations (LSEs). In doing so, we find that LSEs transport free-volume, coordination, and bondorder on-average conservatively between the end points of the displacement sequence whose stringlike geometry arises naturally from the underlying disclination network characterizing the frustrated amorphous structure. Moreover, the average conservative transport of free-volume gives a natural origin to the observed far-field quadrapolarlike shear stress signature admitted by the LSE. Together, these results provide the basis for future coarse-grained models of thermally activated glass plasticity in which the same underlying processes are responsible for material transport and heterogeneous structural evolution, paving the way for a simulation-based quantitative understanding of the bulk metallic glass structure-property relation.

\section{ACKNOWLEDGMENTS}

The present work was supported by the Swiss National Science Foundation under Grant No. 200021-165527.
[1] W. Kauzmann, Chem. Rev. 43, 219 (1948).

[2] D. Chandler and J. P. Garrahan, Annu. Rev. Phys. Chem. 61, 191 (2010).

[3] Y. Sun, A. Concustell, and A. L. Greer, Nat. Rev. Mater. 1, 16039 (2016).

[4] T. C. Hufnagel, C. A. Schuh, and M. L. Falk, Acta Mater. 109, 375 (2016).

[5] M. H. Cohen and D. Turnbull, J. Chem. Phys. 31, 1164 (1959).

[6] D. Turnbull and M. H. Cohen, J. Chem. Phys. 34, 120 (1961).

[7] F. Spaepen, Acta Metal. 25, 407 (1977).

[8] A. S. Argon, Acta Metal. 27, 47 (1979).

[9] D. Rodney, A. Tanguy, and D. Vandembroucq, Model. Simul. Mater. Sci. Eng. 19, 083001 (2011).

[10] M. L. Falk and J. S. Langer, Phy. Rev. E 57, 7192 (1998).

[11] M. L. Falk and J. S. Langer, Annu. Rev. Condens. Matter. Phys. 2, 353 (2011).

[12] S. Sanyal and A. K. Sood, Europhys. Lett. 34, 361 (1996).

[13] H. R. Schober, C. Gaukel, and C. Oligschleger, Prog. Theor. Phys. Suppl. 126, 67 (1997).

[14] C. Oligschleger and H. R. Schober, Phys. Rev. B 59, 811 (1999).

[15] H. Teichler, J. Non Cryst. Solids 293-295, 339 (2001).

[16] F. Faupel, W. Frank, M. P. Macht, H. Mehrer, V. Naundorf, K. Rätzke, H. R. Schober, S. K. Sharma, and H. Teichler, Rev. Mod. Phys. 75, 237 (2003).

[17] P. M. Derlet, Phys. Rev. Materials 4, 125601 (2020).

[18] C. Donati, J. F. Douglas, W. Kob, S. J. Plimpton, P. H. Poole, and S. C. Glotzer, Phys. Rev. Lett. 80, 2338 (1998).

[19] T. B. Schrøder, S. Sastry, J. C. Dyre, and S. C. Glotzer, J. Chem. Phys. 112, 9834 (2000).

[20] Y. Gebremichael, M. Vogel, and S. C. Glotzer, J. Chem. Phys. 120, 4415 (2004).

[21] M. Vogel, B. Doliwa, A. Heuer, and S. C. Glotzer, J. Chem. Phys. 120, 4404 (2004).

[22] T. Kawasaki and A. Onuki, J. Chem. Phys. 138, 12A514 (2013).

[23] D. Rodney and C. A. Schuh, Phys. Rev. Lett. 102, 235503 (2009); Phys. Rev. B 80, 184203 (2009).

[24] S. Swayamjyoti, J. F. Löffler, and P. M. Derlet, Phys. Rev. B 89, 224201 (2014).

[25] S. Swayamjyoti, J. F. Löffler, and P. M. Derlet, Phys. Rev. B 93, 144202 (2016).

[26] Y. Fan, T. Iwashita, and T. Egami, Nat. Commun. 8, 15417 (2017).

[27] P. Koziatek, J.-L. Barrat, P. M. Derlet, and D. Rodney, Phys. Rev. B 87, 224105 (2013).

[28] P. M. Derlet and R. Maass, J. Mat. Res. 32, 2668 (2017).

[29] P. M. Derlet and R. Maass, J. Alloys Comps. 821, 153209 (2020).
[30] P. M. Derlet and R. Maass, Acta Mater. 143, 205 (2018).

[31] P. M. Derlet and R. Maass, Acta. Mater. 209, 116771 (2021).

[32] H. Jónsson and H. C. Andersen, Phys. Rev. Lett. 60, 2295 (1988).

[33] H. W. Sheng, W. K. Luo, F. M. Alamgir, J. M. Bai, and E. Ma, Nature (London) 439, 419 (2006).

[34] E. Ma, Nat. Mater. 14, 547 (2015).

[35] H. Tanaka, H. Tong, R. Shi, and J. Russo, Nat. Rev. Phys. 1, 333 (2019).

[36] F. C. Frank, Proc. R. Soc. London Ser. A 215, 43 (1952).

[37] J. D. Bernal, Proc. R. Soc. London Ser. A 280, 299 (1964).

[38] P. Chaudhari and D. Turnbull, Science 199, 11 (1978).

[39] D. R. Nelson, Phys. Rev. Lett. 50, 982 (1983).

[40] D. R. Nelson, Phys. Rev. B 28, 5515 (1983).

[41] J. D. Eshelby, Proc. R. Soc. London Ser. A 241, 376 (1957).

[42] P. M. Derlet and R. Maass, Philos. Mag. 94, 2776 (2014).

[43] P. M. Derlet and R. Maass, Philos. Mag. 93, 4232 (2013).

[44] S. Nell, F. Yang, Z. Evenson, and A. Meyer, Phys. Rev. B 103, 064206 (2021).

[45] H. R. Schober and H. L. Peng, Phys. Rev. E 93, 052607 (2016).

[46] G. Wahnström, Phys. Rev. A 44, 3752 (1991).

[47] See GitHub repository at https://github.com/derlet/SU2.

[48] C. E. Maloney and A. Lemaitre, Phys. Rev. Lett. 93, 016001 (2004).

[49] K. E. Jensen, D. A. Weitz, and F. Spaepen, Phys. Rev. E 90, 042305 (2014).

[50] V. V. Bulatov and A. S. Argon, Model. Simul. Mater. Sci. Eng. 2, 167 (1994); 2, 185 (1994); 2, 203 (1994).

[51] C. Su and L. Anand, Acta Mater. 54, 179 (2006).

[52] L. Anand and C. Su, Acta Mater. 55, 3735 (2007).

[53] E. R. Homer and C. A. Schuh, Acta Mater. 57, 2823 (2009); Model. Simul. Mater. Sci. Eng. 18, 065009 (2010).

[54] L. Li, E. R. Homer, and C. A. Schuh, Acta Mater. 61, 3347 (2013).

[55] J. Ding, Y.-Q. Cheng, and E. Ma, Acta Mater. 69, 343 (2014).

[56] J. Ding and E. Ma, npj Comput. Mater. 3, 9 (2017).

[57] F. C. Frank and J. S. Kasper, Acta Crystallogr. 11, 184 (1958).

[58] J. Zemp, M. Celino, B. Schönfeld, and J. F. Löffler, Phys. Rev. B. 90, 144108 (2014).

[59] J. Zemp, M. Celino, B. Schönfeld, and J. F. Löffler, Phys. Rev. Lett. 115, 165501 (2015).

[60] C. Liu and R. Maass, Adv. Func. Mater. 28, 1800388 (2018).

[61] J. C. Qiao, Q. Wang, J. M. Pelletier, H. Kato, R. Casalini, D. Crespo, E. Pineda, Y. Yao, and Y. Yang, Prog. Mater. Sci. 104, 250 (2019).

[62] N. A. Mauro, M. Blodgett, M. L. Johnson, A. J. Vogt, and K. F. Kelton, Nat. Commun. 5, 4616 (2014). 